\title{
Particle production in relativistic heavy-ion collisions: A consistent hydrodynamic approach
}

\author{
Rajeev S. Bhalerao, Amaresh Jaiswal, Subrata Pal and V. Sreekanth \\ Tata Institute of Fundamental Research, Homi Bhabha Road, Mumbai 400005, India
}

(Dated: November 12, 2018)

\begin{abstract}
We derive relativistic viscous hydrodynamic equations invoking the generalized second law of thermodynamics for two different forms of the non-equilibrium single-particle distribution function. We find that the relaxation times in these two derivations are identical for shear viscosity but different for bulk viscosity. These equations are used to study thermal dilepton and hadron spectra within longitudinal scaling expansion of the matter formed in relativistic heavy-ion collisions. For consistency, the same non-equilibrium distribution function is used in the particle production prescription as in the derivation of the viscous evolution equations. Appreciable differences are found in the particle production rates corresponding to the two non-equilibrium distribution functions. We emphasize that an inconsistent treatment of the non-equilibrium effects influences the particle production significantly, which may affect the extraction of transport properties of quark-gluon plasma.

PACS numbers: $25.75 .-\mathrm{q}, 24.10 . \mathrm{Nz}, 47.75+\mathrm{f}$
\end{abstract}

\section{INTRODUCTION}

Evolution of the strongly-interacting matter produced in high-energy heavy-ion collisions, when the system is close to local thermodynamic equilibrium, has been studied extensively within the framework of the relativistic dissipative hydrodynamics; for a recent review see Ref. [1]. As the system expands and becomes dilute enough, the hydrodynamic description breaks down, leading to a freezeout or a transition from the hydrodynamic description to a particle description [2]. The dissipative effects are important not only during the hydrodynamic evolution, but also in the particle production [3], and the two have to be treated in a consistent manner. Moreover, the transport coefficients and relaxation times which constitute an external input to the hydrodynamic equations need to be in conformity with the theoretical framework used to derive the hydrodynamic equations [4]. Ad hoc choices or inconsistent treatments could significantly affect the final-state particle yields, spectra, and other observables derived from them.

Hydrodynamics is formulated as an order-by-order expansion in gradients of the hydrodynamic four-velocity $u^{\mu}$. The ideal hydrodynamics is zeroth order and relativistic Navier-Stokes theory is first order in gradients; the latter violates causality. Derivation of the (causal) second-order dissipative hydrodynamic equations proceeds in a variety of ways; for a review see Ref. 5]. For instance, in the derivations based on kinetic theory the non-equilibrium phase-space distribution function $f(x, p)$ needs to be specified. This is commonly achieved by taking recourse to Grad's 14-moment approximation [6]. The hydrodynamic equations are then derived by suitable coarse-graining of the microscopic dynamics. For consistency, the same $f(x, p)$ ought to be used in the particle-production prescription [2, 7] as well. This important consideration has been overlooked in several hydrodynamic studies of heavy-ion collisions.
An alternate derivation of hydrodynamic equations starts from a generalized entropy four-current $S^{\mu}$, expressed in terms of a few unknown coefficients, and then invokes the second law of thermodynamics $\left(\partial_{\mu} S^{\mu} \geq 0\right)$ 5]. These coefficients which are related to relaxation times for shear and bulk pressures remain undetermined, and have to be obtained from kinetic theory [8, 9]. Even then the bulk relaxation time remains ambiguous. Ideally, a single theoretical framework should give rise to dissipative evolution equations as well as determine these unknown coefficients [4]. The bulk relaxation time obtained in Ref. [4] exhibits critical slowing down near the QCD phase transition and does not lead to cavitation.

In this paper, we employ the method based on the entropy four-current to derive second-order viscous hydrodynamics corresponding to two different forms of the non-equilibrium distribution function. These distribution functions are formally different, and one of them is used here for the first time to study the particle production in heavy-ion collisions. We perform a comparative numerical study of these two formalisms in the Bjorken scaling expansion. For consistency, we use the same nonequilibrium distribution function in the calculation of the particle spectra as in the derivation of the evolution equations, and point out drawbacks of an inconsistent treatment. As an application, we study the production of thermal dileptons and hadrons in various scenarios.

The paper is organized as follows. In Sec. II, we derive viscous hydrodynamic equations corresponding to two choices of the non-equilibrium distribution function. In Sec. III we present thermal dilepton and hadron production rates. In Sec. IV we consider the Bjorken scenario and obtain the relevant evolution equations and particle production rates in the two cases. Numerical results are presented and discussed in Sec. V, which is followed by the Summary in Sec. VI. 


\section{VISCOUS HYDRODYNAMICS}

The entropy four-current for particles obeying the Boltzmann statistics is given by [10]

$$
S^{\mu}(x)=-\int d p p^{\mu} f(\ln f-1),
$$

where $d p=g d \mathbf{p} /\left[(2 \pi)^{3} \sqrt{\mathbf{p}^{2}+m^{2}}\right], g$ and $m$ being the degeneracy factor and the particle rest mass, respectively, $p^{\mu}$ is the particle four-momentum, and $f \equiv f(x, p)$ is the single-particle phase-space distribution function. For a system close to equilibrium, $f$ can be written as $f=f_{0}+\delta f \equiv f_{0}(1+\phi)$, where the equilibrium distribution function is defined as $f_{0}=\exp (-\beta u \cdot p)$. Here $\beta \equiv 1 / T$ is the inverse temperature, $u^{\mu}$ is defined in the Landau frame [10], and we have assumed the baryochemical potential to be zero.

The divergence of $S^{\mu}$ reads

$$
\begin{aligned}
\partial_{\mu} S^{\mu} & =-\int d p p^{\mu}\left(\partial_{\mu} f\right) \ln f \\
& =-\int d p p^{\mu}\left[\phi(1+\phi / 2)\left(\partial_{\mu} f_{0}\right)+\phi\left(\partial_{\mu} \phi\right) f_{0}\right],
\end{aligned}
$$

where in the second equality terms up to third order in gradients have been retained.

To proceed further, the non-equilibrium part of the distribution function $\delta f \equiv f_{0} \phi$ needs to be specified. In the present study, we consider two different forms of $\phi$. The first form is consistent with Grad's 14-moment approximation [6] for the single-particle distribution function in orthogonal basis [1]. We propose [4]

$$
\phi_{1}=\frac{\Pi}{P}+\frac{p^{\mu} p^{\nu} \pi_{\mu \nu}}{2(\epsilon+P) T^{2}},
$$

where corrections up to second order in momenta are present. Equation (3) has not been used before to study particle production in heavy-ion collisions. The second form is obtained by considering the corrections which are only quadratic in momenta [12]:

$$
\phi_{2}=\frac{p^{\mu} p^{\nu}}{2(\epsilon+P) T^{2}}\left(\pi_{\mu \nu}+\frac{2}{5} \Pi \Delta_{\mu \nu}\right) .
$$

In Eqs. (3) and (44), $\epsilon$ and $P$ are the thermodynamic energy density and pressure, respectively, $\Pi$ the bulk viscous pressure, $\pi^{\mu \nu}$ the shear stress tensor, and $\Delta^{\mu \nu}=$ $g^{\mu \nu}-u^{\mu} u^{\nu}$. The energy-momentum tensor can be expressed in terms of these quantities as $T^{\mu \nu}=\epsilon u^{\mu} u^{\nu}-$ $(P+\Pi) \Delta^{\mu \nu}+\pi^{\mu \nu}$. Note that although the contributions due to shear in Eqs. (3) and (4) are identical, those due to bulk viscosity are distinct. In the following, we shall refer to analyses performed using Eqs. (31) and (4) as 'Case 1' and 'Case 2', respectively.

Performing the integrals in Eq. (2) as outlined in Ref.
[4], we obtain

$$
\begin{aligned}
\partial_{\mu} S^{\mu}= & -\beta \Pi\left[\theta+\beta_{0} \dot{\Pi}+\frac{4}{3} \beta_{0} \theta \Pi\right] \\
& +\beta \pi^{\mu \nu}\left[\sigma_{\mu \nu}-\beta_{2} \dot{\pi}_{\mu \nu}-\frac{4}{3} \beta_{2} \theta \pi_{\mu \nu}\right],
\end{aligned}
$$

where $\beta_{0}$ and $\beta_{2}$ are functions of thermodynamic quantities $\epsilon$ and $T, \dot{X} \equiv u^{\mu} \partial_{\mu} X, \theta=\partial_{\mu} u^{\mu}$, and $\sigma^{\mu \nu}=\nabla^{\langle\mu} u^{\nu\rangle}$. The notation $A^{\langle\mu \nu\rangle}=\Delta_{\alpha \beta}^{\mu \nu} A^{\alpha \beta}$, where $\Delta_{\alpha \beta}^{\mu \nu}=\left[\Delta_{\alpha}^{\mu} \Delta_{\beta}^{\nu}+\right.$ $\left.\Delta_{\beta}^{\mu} \Delta_{\alpha}^{\nu}-(2 / 3) \Delta^{\mu \nu} \Delta_{\alpha \beta}\right] / 2$, represents the traceless symmetric projection orthogonal to $u^{\mu}$.

The second law of thermodynamics, $\partial_{\mu} S^{\mu} \geq 0$, is guaranteed to be satisfied if linear relationships between thermodynamical fluxes and extended thermodynamic forces are imposed. This leads to the following evolution equations for bulk and shear

$$
\begin{aligned}
\Pi & =-\zeta\left[\theta+\beta_{0} \dot{\Pi}+\frac{4}{3} \beta_{0} \theta \Pi\right], \\
\pi^{\mu \nu} & =2 \eta\left[\sigma^{\mu \nu}-\beta_{2} \dot{\pi}^{\langle\mu \nu\rangle}-\frac{4}{3} \beta_{2} \theta \pi^{\mu \nu}\right],
\end{aligned}
$$

respectively, where the coefficients of bulk and shear viscosity satisfy $\zeta, \eta \geq 0$. The bulk and shear relaxation times, defined as $\tau_{\Pi}=\zeta \beta_{0}$ and $\tau_{\pi}=2 \eta \beta_{2}$, can be obtained directly from the transport coefficients $\beta_{0}$ and $\beta_{2}$ which are determined explicitly in the above derivations.

For Case 1 , the coefficients $\beta_{0}$ and $\beta_{2}$ become

$$
\beta_{0}^{(1)}=1 / P, \quad \beta_{2}^{(1)}=3 /(\epsilon+P)+m^{2} \beta^{2} P /\left[2(\epsilon+P)^{2}\right],
$$

whereas for Case 2, they reduce to

$$
\beta_{0}^{(2)}=\frac{18}{5(\epsilon+P)}+\frac{3 m^{2} \beta^{2} P}{5(\epsilon+P)^{2}}, \quad \beta_{2}^{(2)}=\beta_{2}^{(1)} .
$$

We note that although the relaxation time corresponding to shear $\left(\beta_{2}\right)$ is the same for both the cases, that corresponding to bulk $\left(\beta_{0}\right)$ is different. We stress that these coefficients have been obtained consistently within a single theoretical framework. This is in contrast to the standard derivation [8], where the transport coefficients have to be estimated from an alternate theory.

\section{THERMAL DILEPTON AND HADRON PRODUCTION}

Particle production is influenced by viscosity in two ways: first through the viscous hydrodynamic evolution of the system and second through corrections to the particle production rate via the non-equilibrium distribution function [3]. Hydrodynamic evolution was considered in the previous section; here we will concentrate on the thermal dilepton and hadron production rates in heavy-ion collisions. While the hadrons are emitted mostly in the final stages of the evolution, the dileptons are emitted at 
all stages and thus probe the entire temperature history of the system.

In the quark-gluon plasma (QGP) phase, the dominant production mechanism for dileptons is $q \bar{q} \rightarrow \gamma^{*} \rightarrow l^{+} l^{-}$, whereas in the hadronic phase the main contribution arises from $\pi^{+} \pi^{-} \rightarrow \rho^{0} \rightarrow l^{+} l^{-}$. The dilepton production rate for these processes is given by [13]

$$
\begin{aligned}
\frac{d N}{d^{4} x d^{4} p}=g^{2} \int & \frac{d^{3} \mathbf{p}_{1}}{(2 \pi)^{3}} \frac{d^{3} \mathbf{p}_{2}}{(2 \pi)^{3}} f\left(E_{1}, T\right) f\left(E_{2}, T\right) \\
& \times v_{r e l} \sigma\left(M^{2}\right) \delta^{4}\left(p-p_{1}-p_{2}\right),
\end{aligned}
$$

where $p_{i}=\left(E_{i}, \mathbf{p}_{i}\right)$ are the four-momenta of the incoming particles having equal masses $m_{i}$ and relative velocity $v_{r e l}=M\left(M^{2}-4 m_{i}^{2}\right)^{1 / 2} / 2 E_{1} E_{2}$. Further, $M$ and $\sigma\left(M^{2}\right)$ are the dilepton invariant mass and production cross section, respectively. Substituting for $f=f_{0}+\delta f$ and retaining only the terms linear in $\delta f$, the dilepton production rate can be expressed as a sum of contributions due to ideal, shear, and bulk:

$$
\frac{d N}{d^{4} x d^{4} p}=\frac{d N^{(0)}}{d^{4} x d^{4} p}+\frac{d N^{(\pi)}}{d^{4} x d^{4} p}+\frac{d N^{(\Pi)}}{d^{4} x d^{4} p} .
$$

For the case $M \gg T \gg m_{i}$, the equilibrium distribution functions can be approximated by the MaxwellBoltzmann form $f(E, T)=\exp (-E / T)$ and $v_{r e l} \simeq$ $M^{2} / 2 E_{1} E_{2}$. In the QGP phase (for $q \bar{q}$ annihilation) we have $M^{2} g^{2} \sigma\left(M^{2}\right)=(80 \pi / 9) \alpha^{2}$ (with $N_{f}=2$ and $N_{c}=3$ ) and in the hadronic phase (for $\pi^{+} \pi^{-}$annihilation) we have $M^{2} g^{2} \sigma\left(M^{2}\right)=(4 \pi / 3) \alpha^{2}\left|F_{\pi}\left(M^{2}\right)\right|^{2}$ [13]. The electromagnetic pion form factor is $\left|F_{\pi}\left(M^{2}\right)\right|^{2}=$ $m_{\rho}^{4} /\left[\left(m_{\rho}^{2}-M^{2}\right)^{2}+m_{\rho}^{2} \Gamma_{\rho}^{2}\right]$, where $m_{\rho}=775 \mathrm{MeV}$ and $\Gamma_{\rho}=149 \mathrm{MeV}$ are the mass and decay width of the $\rho(770)$ meson, respectively [14].

With the above approximations, the integrals in Eq. (11) can be performed. The ideal part is given by [13]

$$
\frac{d N^{(0)}}{d^{4} x d^{4} p}=\frac{1}{2} \frac{M^{2} g^{2} \sigma\left(M^{2}\right)}{(2 \pi)^{5}} e^{-p_{0} / T} .
$$

The shear viscosity contribution is the same for $\phi_{1}$ and $\phi_{2}$, Eqs. (3) and (4), and is given by [15]

$$
\frac{d N^{(\pi)}}{d^{4} x d^{4} p}=\frac{2}{3}\left(\frac{p^{\mu} p^{\nu}}{2 s T^{3}} \pi_{\mu \nu}\right) \frac{d N^{(0)}}{d^{4} x d^{4} p}
$$

where $s=(\epsilon+P) / T$ is the equilibrium entropy density. The bulk viscosity contribution for $\phi_{1}$ is

$$
\frac{d N_{1}^{(\Pi)}}{d^{4} x d^{4} p}=\frac{\Pi}{P} \frac{d N^{(0)}}{d^{4} x d^{4} p},
$$

and that for $\phi_{2}$ can be expressed as [16]

$$
\frac{d N_{2}^{(\Pi)}}{d^{4} x d^{4} p}=\frac{2}{5 s T^{3}}\left(\frac{M^{2}}{12} g^{\alpha \beta}-\frac{1}{3} p^{\alpha} p^{\beta}\right) \Delta_{\alpha \beta} \Pi \frac{d N^{(0)}}{d^{4} x d^{4} p} .
$$

The hadron spectra are obtained using the CooperFrye freezeout prescription [2]

$$
\frac{d N}{d^{2} p_{T} d y}=\frac{g}{(2 \pi)^{3}} \int p_{\mu} d \Sigma^{\mu} f(x, p),
$$

where $d \Sigma^{\mu}$ represents the element of the threedimensional freezeout hypersurface, and $f(x, p)$ represents the phase-space distribution function at freezeout.

For the two cases discussed above we shall study the evolution of the hydrodynamic variables and their influence on the dilepton and hadron production rates.

\section{BJORKEN SCENARIO}

We consider the evolution of the system in longitudinal scaling expansion at vanishing net baryon number density [17]. In terms of the Milne coordinates $\left(\tau, r, \varphi, \eta_{s}\right)$, where $\tau=\sqrt{t^{2}-z^{2}}$ and $\eta_{s}=\tanh ^{-1}(z / t)$, and with $u^{\mu}=(1,0,0,0)$, evolution equations for $\epsilon, \Phi \equiv-\tau^{2} \pi^{\eta_{s} \eta_{s}}$, and $\Pi$ become

$$
\begin{aligned}
\frac{d \epsilon}{d \tau} & =-\frac{1}{\tau}(\epsilon+P+\Pi-\Phi), \\
\tau_{\pi} \frac{d \Phi}{d \tau} & =\frac{4 \eta}{3 \tau}-\Phi-\frac{4 \tau_{\pi}}{3 \tau} \Phi, \\
\tau_{\Pi} \frac{d \Pi}{d \tau} & =-\frac{\zeta}{\tau}-\Pi-\frac{4 \tau_{\Pi}}{3 \tau} \Pi .
\end{aligned}
$$

The bulk and shear relaxation times, $\tau_{\Pi}=\zeta \beta_{0}$ and $\tau_{\pi}=$ $2 \eta \beta_{2}$, reduce to

$$
\tau_{\Pi}^{(1)}=\frac{\epsilon+P}{P T}\left(\frac{\zeta}{s}\right), \quad \tau_{\Pi}^{(2)}=\frac{18}{5 T}\left(\frac{\zeta}{s}\right), \quad \tau_{\pi}=\frac{6}{T}\left(\frac{\eta}{s}\right),
$$

for the two different forms of $\phi$, Eqs. (3) and (4).

Once the temperature evolution is known from the hydrodynamical model, the total dilepton spectrum is obtained by integrating the total rate over the space-time evolution of the system

$$
\frac{d N_{1,2}}{d^{2} p_{T} d M^{2} d y}=\pi R_{A}^{2} \int_{\tau_{0}}^{\tau_{f o}} d \tau \tau \int_{-\infty}^{\infty} d \eta_{s}\left(\frac{1}{2} \frac{d N_{1,2}}{d^{4} x d^{4} p}\right),
$$

where $\tau_{0}$ and $\tau_{f o}$ are the initial and freezeout times for the hydrodynamic evolution. We note that for the Bjorken expansion, $d^{4} x=\pi R_{A}^{2} d \eta_{s} \tau d \tau$, where $R_{A}=1.2 A^{1 / 3}$ is the nuclear radius.

In $\left(\tau, r, \varphi, \eta_{s}\right)$ coordinates, particle four-momentum is $p^{\mu}=\left(m_{T} \cosh \left(y-\eta_{s}\right), \quad p_{T} \cos \left(\varphi_{p}-\varphi\right), p_{T} \sin \left(\varphi_{p}-\right.\right.$ $\left.\varphi) / r, m_{T} \sinh \left(y-\eta_{s}\right) / \tau\right)$, where $m_{T}^{2}=p_{T}^{2}+m^{2}$. The other factors appearing in the rate expressions, Eqs. (13) and (15), are then given by

$$
\begin{aligned}
p^{\alpha} p^{\beta} \pi_{\alpha \beta} & =\frac{\Phi}{2} p_{T}^{2}-\Phi m_{T}^{2} \sinh ^{2}\left(y-\eta_{s}\right), \\
p^{\alpha} p^{\beta} \Delta_{\alpha \beta} & =-p_{T}^{2}-m_{T}^{2} \sinh ^{2}\left(y-\eta_{s}\right) .
\end{aligned}
$$


Similar to the dilepton spectra, the hadronic spectra can also be split up into three parts. Writing the momentum flux through the hypersurface element as $p_{\mu} d \Sigma^{\mu}=m_{T} \cosh \left(y-\eta_{s}\right) \tau d \eta_{s} r d r d \varphi$, and performing the $\eta_{s}$ integration, we get for the ideal case,

$$
\frac{d N^{(0)}}{d^{2} p_{T} d y}=\frac{g}{(2 \pi)^{2}} m_{T} \tau_{f o} R_{A}^{2} K_{1}\left(z_{m}\right)
$$

where $K_{n}$ are the modified Bessel functions of the second kind and $z_{m} \equiv m_{T} / T$. The contribution due to the shear viscosity to the hadron production reduces to

$$
\frac{d N^{(\pi)}}{d^{2} p_{T} d y}=\frac{\Phi}{4(\epsilon+P)}\left[z_{p}^{2}-2 z_{m} \frac{K_{2}\left(z_{m}\right)}{K_{1}\left(z_{m}\right)}\right] \frac{d N^{(0)}}{d^{2} p_{T} d y},
$$

where $z_{p} \equiv p_{T} / T$. The bulk viscosity contribution in Case 1, Eq. (3), is calculated to be

$$
\frac{d N_{1}^{(\Pi)}}{d^{2} p_{T} d y}=\frac{\Pi}{P} \frac{d N^{(0)}}{d^{2} p_{T} d y},
$$

whereas in Case 2, Eq. (44), it reduces to

$$
\frac{d N_{2}^{(\Pi)}}{d^{2} p_{T} d y}=\frac{-\Pi}{5(\epsilon+P)}\left[z_{p}^{2}+z_{m} \frac{K_{2}\left(z_{m}\right)}{K_{1}\left(z_{m}\right)}\right] \frac{d N^{(0)}}{d^{2} p_{T} d y} .
$$

Here we have used the recurrence relation $K_{n+1}(z)=$ $2 n K_{n}(z) / z+K_{n-1}(z)$. It is important to note that the bulk viscosity contribution in Case 1 is negative, whereas that in Case 2 is positive $(\Pi<0)$.

\section{NUMERICAL RESULTS AND DISCUSSION}

We now present numerical results for the Bjorken expansion of the medium for the initial temperature $T_{0}=$ $310 \mathrm{MeV}$ and time $\tau_{0}=0.5 \mathrm{fm} / \mathrm{c}$, typical for the Relativistic Heavy-Ion Collider. The freezeout temperature was taken to be $160 \mathrm{MeV}$. The initial pressure configuration was assumed to be isotropic: $\Phi=0=\Pi$. We employ the equation of state of Refs. [18, 19] based on a recent lattice QCD simulation. The shear viscosity to entropy density ratio $\eta / s$ was taken to be $1 / 4 \pi$ corresponding to the conjectured lower bound obtained in Ref. [20]. For the bulk viscosity to entropy density ratio $\zeta / s$ at $T \geq T_{c} \approx 184 \mathrm{MeV}$, we adopted a parametrized form of the lattice QCD result; see Refs. 21, 22]. For $T<T_{c}$, we parametrized $\zeta / s$ given in Ref. 23].

Figure1(a) presents the time evolution of shear $\Phi$ and bulk $\Pi$ viscous pressures for the various bulk relaxation times $\tau_{\Pi}$ defined in Eq. (20). At times $\tau \gtrsim 3 \mathrm{fm} / \mathrm{c}$, corresponding to temperatures $T \lesssim 1.2 T_{c}$, the bulk dominates the shear pressure which can influence the particle production appreciably. The widely used choice $\tau_{\Pi}=\tau_{\pi}$ (dotted-dashed line) leads to vanishing longitudinal pressure $P_{L}$ and cavitation [22] as is evident in Fig. 1(b). On the other hand, $\tau_{\Pi}=\tau_{\Pi}^{(1,2)}$ does not lead to cavitation

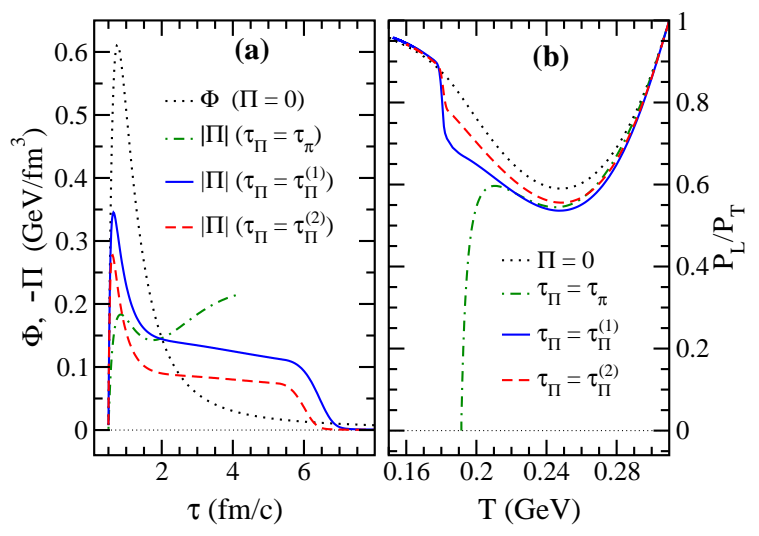

FIG. 1: (color online) (a) Time evolution of shear $\Phi$ and bulk $\Pi$ viscous pressures, and (b) temperature dependence of the ratio of the longitudinal to transverse pressures $P_{L} / P_{T}$, for the various bulk relaxation times $\tau_{\Pi}$ defined in Eq. (20). Note that for $\tau_{\Pi}=\tau_{\pi}$, cavitation $\left(P_{L}<0\right)$ sets in.

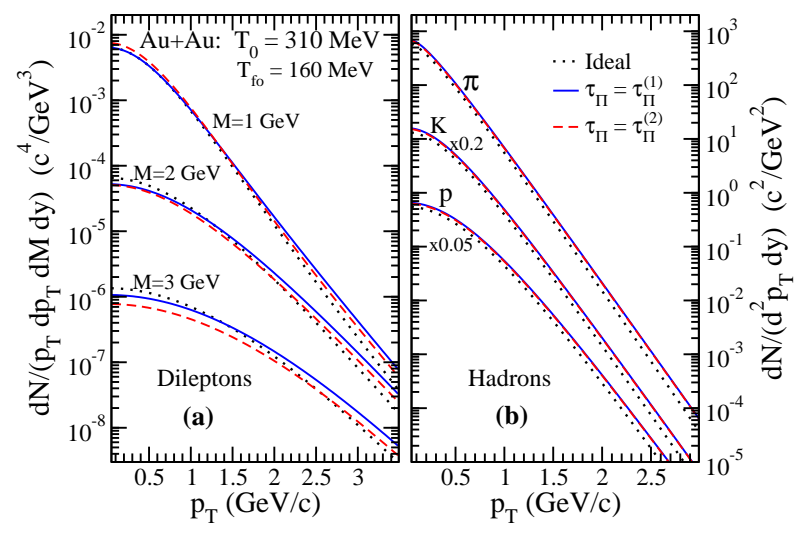

FIG. 2: (color online) Particle spectra as a function of the transverse momentum $p_{T}$, for ideal and viscous hydrodynamics with bulk relaxation times $\tau_{\Pi}$ defined in Eq. (20) for (a) dileptons of invariant mass $M=1,2,3 \mathrm{GeV} / c^{2}$, and (b) hadrons.

as discussed in [4]. As $\tau_{\Pi}^{(1)}>\tau_{\Pi}^{(2)}$ at all times, the magnitude of $\Pi$ is found to be larger in Case 1 (solid line). This leads to enhanced pressure anisotropy, i.e., a larger departure of $P_{L} / P_{T}=(P+\Pi-\Phi) /(P+\Pi+\Phi / 2)$ from unity.

Figure 2 displays dilepton and hadron transverse momentum spectra for the two choices of $\tau_{\Pi}$, in comparison with the ideal hydrodynamic calculation, and Fig. 3 shows the same spectra normalized by the ideal case. Note the enhancement of the dilepton spectra at high $p_{T}$, and their suppression at low $p_{T}$ compared to the ideal case. The high- $p_{T}$ dileptons emerge predominantly at early times when the temperature and density are high. Viscosity slows down the cooling of the system [9] producing a relatively larger number of hard dileptons. We observe that at high $p_{T}$, the viscous correction to the dilepton production rate due to shear is positive and dominates that due to bulk. The low- $p_{T}$ dileptons are 
produced mainly at later stages of the evolution when the negative correction due to the bulk viscosity dominates (Fig. 11) leading to the suppression of the spectra compared to the ideal case. Further for Case 2 [red (light grey lines)], the $p_{T}^{2}$ dependence of the viscous correction, Eqs. (15) and (23), implies a smaller enhancement (suppression) at high (low) $p_{T}$, compared to Case 1 [blue (dark grey lines)]. The $M$-dependent splitting is consistent with Eqs. (14) to (15).

Figure 3(b) shows the pion spectra scaled by the ideal case for the two choices of $\tau_{\Pi}$. The negative contribution from the bulk viscous correction for Case 1, Eq. (26), causes suppression of the ratio relative to Case 2, Eq. (27), where the correction is positive. More massive hadrons display qualitatively similar behavior. Interestingly, at high $p_{T}$, dileptons and hadrons display opposite trends for $\tau_{\Pi}^{(1)}$ and $\tau_{\Pi}^{(2)}$ (Fig. 3).

Finally, Fig. [4shows the dilepton invariant mass spectra for the two cases [Eqs. (3) and (4)] in comparison with the ideal case. Results based on Case 1 (blue solid) are almost the same as those obtained in the ideal case at all invariant masses. This is essentially due to the fact that the invariant mass spectrum is dictated by the yields at small $p_{T}$ where the two are nearly identical (Fig. 2(a)). For Case 2 (red dashed) the spectrum exhibits enhanced low-mass and suppressed high-mass dilepton yields. This again can be traced back to the trend seen in Fig. 2(a).

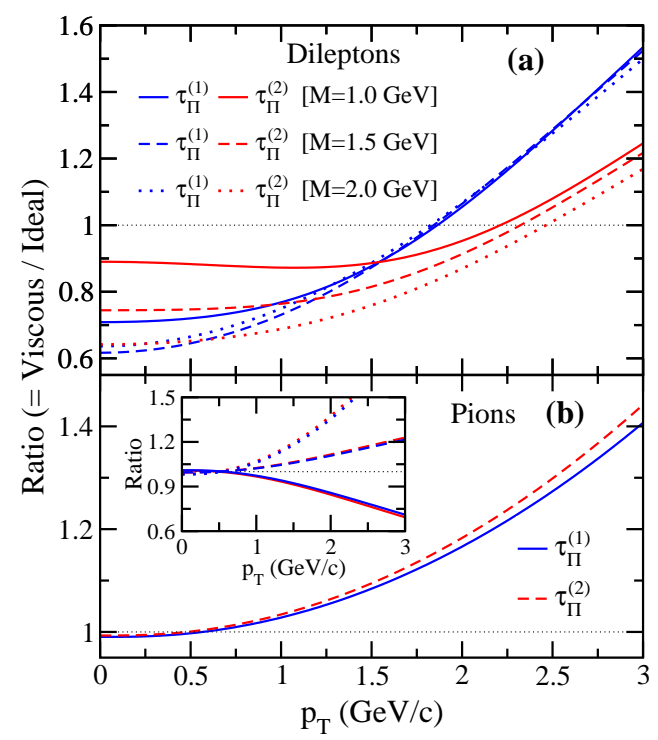

FIG. 3: (color online) Ratios of particle yields for viscous and ideal hydrodynamics as a function of $p_{T}$, for the two bulk relaxation times $\tau_{\Pi}$ defined in Eq. (20) for (a) dileptons of invariant mass $M=1,1.5,2 \mathrm{GeV} / c^{2}$, and (b) pions. Inset: Pion yields in various evolution and production scenarios scaled by the consistent second-order calculation for Case 1 [blue (dark grey)] and Case 2 [red (light grey)]. Solid lines: second-order evolution with ideal production rate; Dashed lines: second-order evolution with first-order correction to the production rate; Dotted lines: ideal evolution with first-order correction to the production rate.

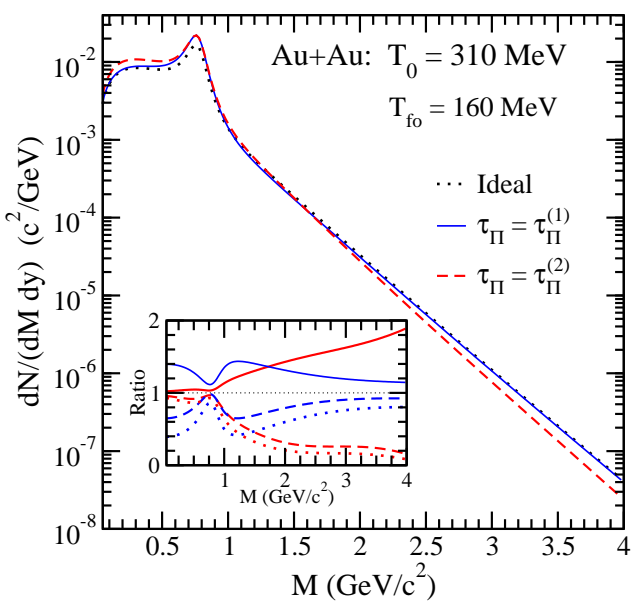

FIG. 4: (color online) Dilepton yields as a function of the invariant mass $M$, in ideal and viscous hydrodynamics with the two bulk relaxation times $\tau_{\Pi}$ defined in Eq. (20). Inset: Same as Fig. 3 inset but for dileptons.

Note that the peak at $M=0.77 \mathrm{GeV} / c^{2}$ corresponds to the dilepton production from the $\rho(770)$ decay.

In contrast to the consistent approach adopted here, in Refs. 15, 24], ideal hydrodynamical evolution was followed by particle production with non-ideal $f(x, p)$ up to first order in gradients. On the other hand, in Refs. [16, 25, 26], although the evolution was according to the second-order viscous hydrodynamics, the freezeout procedure involved ideal [25] or Navier-Stokes [16, 26] corrections to the $f(x, p)$. To illustrate the differences arising due to inconsistent approaches, we show, in the insets of Figs. 3 and 4, pion and dilepton production rates, respectively, in various evolution and production scenarios scaled by the rate obtained in a consistent second-order calculation. We find that the results deviate from unity significantly which may have important implications for the on-going efforts to extract transport properties of QGP within a hydrodynamic framework.

\section{SUMMARY}

Starting with the entropy four-current expressed in terms of the single-particle distribution function, and using two different forms of the correction to the equilibrium distribution function, we derived second-order evolution equations for the viscous dissipative fluxes by invoking the generalized form of the second law of thermodynamics. For consistency, the same non-equilibrium distribution functions were used in the two calculations of particle production. One of the two forms (Eq. (3)) has been used here for the first time to study particle production. In the Bjorken scaling expansion, appreciable differences were found in the two sets of results.

More importantly, we also compared results of various inconsistent calculations with those of a consistent second-order calculation; see insets in Figs. 3 and 4 . It 
is clear that the particle yields are significantly affected if the viscous effects in hydrodynamic evolution and particle production are not mutually consistent. There is no a priori reason to believe that in realistic $2+1$ or $3+1$ dimensional calculations this will not be the case. It will then have important consequences for the extrac- tion of transport properties of the quark-gluon plasma in the hydrodynamic scenario. In conclusion, the nonequilibrium distribution function used in the particle production should be the same as that used in the derivation of hydrodynamic evolution equations.
[1] U. W. Heinz and R. Snellings, Annu. Rev. Nucl. Part. Sci. 63, 123 (2013).

[2] F. Cooper and G. Frye, Phys. Rev. D 10, 186 (1974).

[3] D. Teaney, Phys. Rev. C 68, 034913 (2003).

[4] A. Jaiswal, R. S. Bhalerao and S. Pal, Phys. Rev. C 87, 021901(R) (2013).

[5] P. Romatschke, Int. J. Mod. Phys. E 19, 1 (2010).

[6] H. Grad, Comm. Pure Appl. Math. 2, 331 (1949).

[7] L. D. McLerran and T. Toimela, Phys. Rev. D 31, 545 (1985).

[8] W. Israel and J. M. Stewart, Ann. Phys. (NY) 118, 341 (1979).

[9] A. Muronga, Phys. Rev. C 69, 034903 (2004).

[10] S.R. de Groot, W.A. van Leeuwen, and Ch.G. van Weert, Relativistic Kinetic Theory - Principles and Applications (North-Holland, Amsterdam, 1980).

[11] G. S. Denicol, H. Niemi, E. Molnar and D. H. Rischke, Phys. Rev. D 85, 114047 (2012).

[12] K. Dusling and D. Teaney, Phys. Rev. C 77, 034905 (2008).

[13] R. Vogt, Ultrarelativistic Heavy-Ion Collisions, (NorthHolland, Amsterdam, 2007).

[14] T. Song, K. C. Han and C. M. Ko, Phys. Rev. C 83,
024904 (2011).

[15] K. Dusling and S. Lin, Nucl. Phys. A 809, 246 (2008).

[16] J. R. Bhatt, H. Mishra and V. Sreekanth, Nucl. Phys. A 875, 181 (2012).

[17] J. D. Bjorken, Phys. Rev. D 27, 140 (1983).

[18] P. Huovinen and P. Petreczky, Nucl. Phys. A 837, 26 (2010).

[19] A. Bazavov et al., Phys. Rev. D 80, 014504 (2009).

[20] P. Kovtun, D. T. Son and A. O. Starinets, Phys. Rev. Lett. 94, 111601 (2005).

[21] H. B. Meyer, Phys. Rev. Lett. 100, 162001 (2008).

[22] K. Rajagopal and N. Tripuraneni, JHEP 1003, 018 (2010).

[23] M. Prakash, M. Prakash, R. Venugopalan and G. Welke, Phys. Rept. 227, 321 (1993).

[24] A. Monnai and T. Hirano, Phys. Rev. C 80, 054906 (2009).

[25] G. S. Denicol, T. Kodama, T. Koide and P. Mota, Phys. Rev. C 80, 064901 (2009).

[26] K. Dusling and T. Schaefer, Phys. Rev. C 85, 044909 (2012). 